\title{
Esophageal Adenocarcinoma Arising from Barrett's Dysplasia: A Case Report of Double Occurrence and Prolonged Survival after Chemotherapy
}

\author{
Hemender S. Vats, MD; Tarit K. Banerjee, MD, FACP; Jeffrey Resnick, MD; and Qaseem Khan, MD
}

\begin{abstract}
A relatively young patient with chronic gastroesophageal reflux disease (GERD), obesity, smoking, and alcohol intake presented with widespread metastatic disease in lymph nodes, liver and lungs from a lower esophageal adenocarcinoma extending into the gastroesophageal junction associated with Barrett's mucosa and dysplasia.A complete response was achieved with six cycles of chemotherapy that sustained for more than 4 years without further recurrence. Unfortunately, there was presence of esophageal metaplasia after complete response which eventually converted to low to high grade dysplasia and ultimately to a second primary localized lower esophageal adenocarcinoma that was treated with thoracoabdominal esophagectomy and lymphadenectomy. No evidence of disease recurrence was seen 2 years later. The pathogenesis of a recent increase in the incidence of GERD, Barrett's esophagus and lower esophageal adenocarcinoma are discussed. Surgery, radiotherapy and combination chemotherapy are effective in the early stages leading to tumor shrinkage and prolongation of life and even cure in some cases. Lower esophageal adenocarcinoma is frequently associated with Barrett's high-grade dysplasia. Since there has been a dramatic increase in the incidence of Barrett's dysplasia, appropriate surveillance with upper gastrointestinal endoscopy and preventive strategies, such as the use of aspirin, cyclo-oxygenase II inhibitors and other nonsteroidal antiinflammatory drugs known to be chemopreventive agents against colon, esophagus, gastric and bladder cancers, need to be studied.
\end{abstract}

Keywords: Barrett's esophagus; Dysplasia; Esophageal adenocarcinoma; Gastroesophageal reflux disease

Reprint Requests:

Hemender Singh Vats, MD

Department of General Internal Medicine

Marshfield Clinic

1000 North Oak Avenue

Marshfield, WI 54449

Tel: 715-387-5537

Fax: 7/5-389-3808

Email: vats.hemender@marshfieldclinic.org
Received: May 5, 2006

Revised: July 19, 2006

Accepted: July 26, 2006
E

sophageal adenocarcinoma is now the most common type of esophageal cancer in the United States. Barrett's esophagus and subsequent development of dysplastic changes increase its risk of conversion to adenocarcinoma. Frequent monitoring with endoscopic biopsies can detect adenocarcinoma in the very early stages where treatment can be curative. We report an interesting case where an initial metastatic disease had an excellent response to chemotherapy but later another adenocarcinoma developed from the persistent Barrett's dysplasia.

\section{Case Report}

A 47-year-old Caucasian man presented with left sided supra-clavicular lymphadenopathy. His other medical problems included hypertension, gout, morbid obesity, obstructive sleep apnea and a long-standing history of gastroesophageal reflux symptoms. He denied any history of dysphagia, weight loss or loss of appetite. He was a farmer and had smoked heavily in the past with decreased smoking for the past 10 years. He had moderate alcohol consumption 

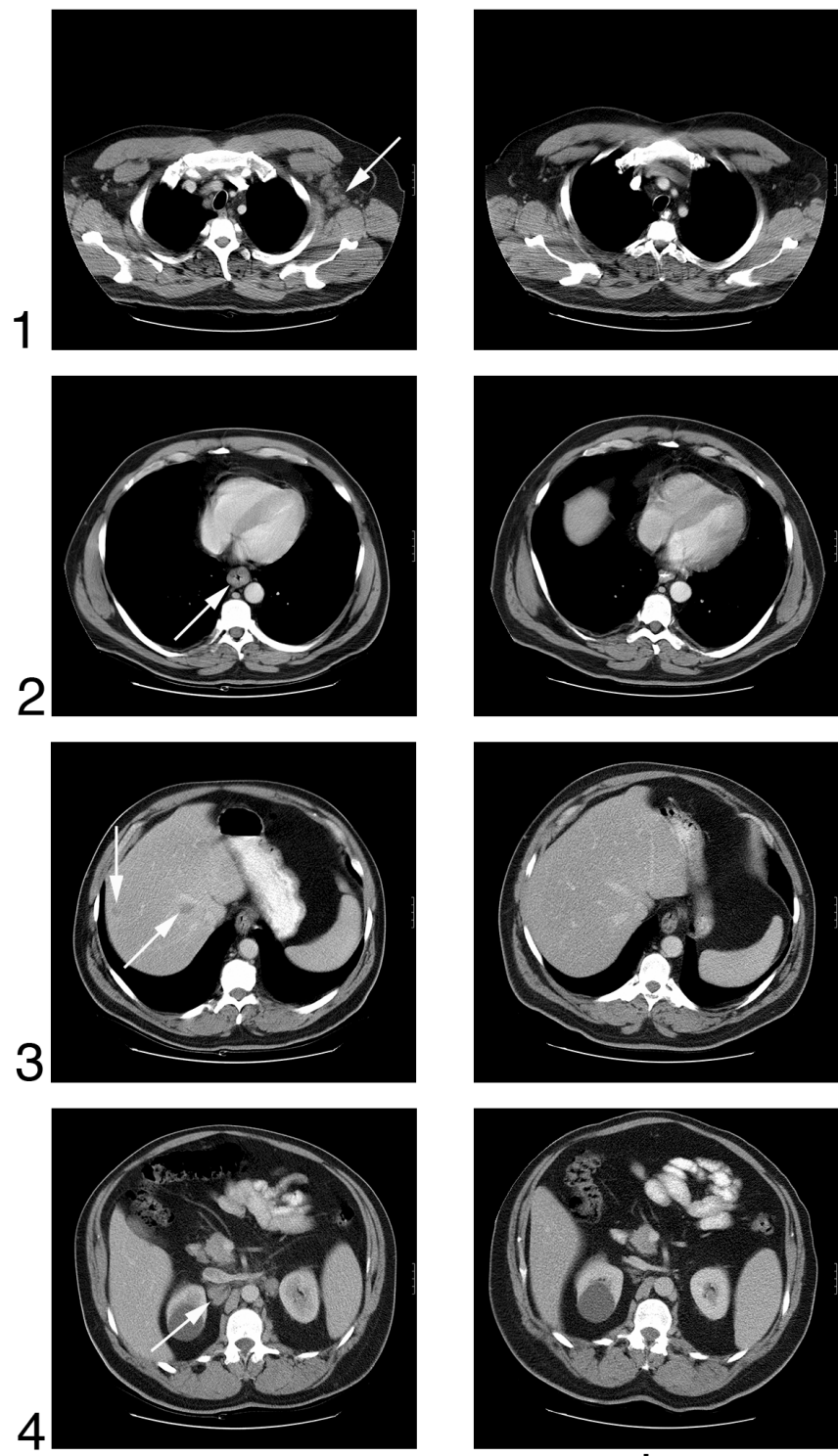

a

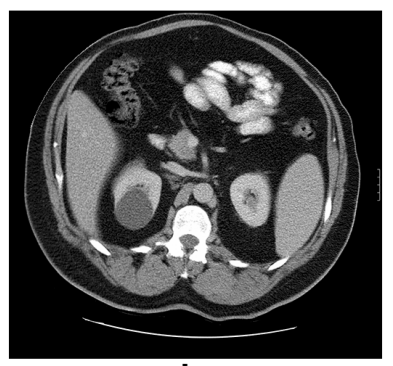

b

Figure 1. Computed tomographic (CT) images of chest and abdomen in (column a) prechemotherapy and (column b) postchemotherapy period (5 months after initial diagnosis) demonstrating the excellent response to chemotherapy. Row 1, Axillary lymphadenopathy. Row 2, Lower esophageal thickening. Row 3, Liver metastases. Row 4, Periaortic lymphadenopathy. Subsequent CT scans did not reveal any evidence of visceral or lymph node malignancy.

in his youth. There was a history of breast cancer in his mother. His medications included allopurinol, losartan and diltiazem.

A biopsy of the supraclavicular lymph node revealed metastatic large cell carcinoma, suggestive of adenocarcinoma. Detailed evaluation for the primary source with computed tomography (CT) scans of the chest, abdomen and pelvis revealed abnormal thickening of the lower esophageal wall and extensive lymphadenopathy in the left axillary, left supraclavicular, perihilar, hepatogastric, perigastric, celiac, superior mesenteric and periaortic regions.

There was also evidence of hepatic metastases along with a couple of right lung metastases (figure 1, column a). Esophagogastroduodenoscopy (EGD) revealed a friable ulcerated exophytic growth $5 \mathrm{~cm}$ in length in the lower third of the esophagus extending into the gastroesophageal junction. The proximal and mid esophagus, stomach and duodenum were normal. A biopsy of the mass confirmed poorly differentiated invasive adenocarcinoma along with the presence of Barrett's high-grade dysplasia (figure 2a). The histology of the metastatic lymph node and esophageal mass was similar.

Based on the biopsy findings of the esophageal mass and the imaging studies, a diagnosis of metastatic (stage IV) adenocarcinoma of the lower third of the esophagus was made, and the patient was treated with three cycles of 5-fluorouracil (24-hour infusion daily for 4 days via Hickman catheter and ambulatory pump), cisplatin and paclitaxel (3-hour infusions of each) every 3 weeks. Three additional cycles using carboplatin as 30-minute infusions replacing cisplatin due to poor tolerance were administered. After six cycles of chemotherapy, there was a complete response in measurable disease clinically and radiologically (figure 1, columns a and b). A repeat EGD, after completion of chemotherapy, also demonstrated the complete response endoscopically with presence of a benign nodule $0.5 \mathrm{~cm}$ at the gastroesophageal junction. Biopsy revealed intestinal metaplasia but no dysplasia or malignancy (figure 2b).

The patient underwent surveillance with EGD and CT scans, as follow-up, and there was no evidence of recurrence of malignancy. However, Barrett's esophagus persisted and subsequent biopsies continued to demonstrate dysplastic changes (figure $2 \mathrm{c}$ and $2 \mathrm{~d}$ ). The average length of the Barrett's segment was $5-7 \mathrm{~cm}$. The grading of dysplasia, over time, changed from focal high-grade changes to diffuse high-grade with ulceration. As initial presentation showed diffusely metastatic disease, surgical treatment was not initially considered. After 4 years in complete remission, and with worsening Barrett's dysplastic changes in the lower esophagus, a surgical consultation was obtained. Surgical treatment was not recommended as there was no malignancy. However, photodynamic therapy was considered, but the patient preferred to have further surveillance.

The patient's EGD at 4 years after initial diagnosis demonstrated the presence of Barrett's esophagus along with a mass measuring $4-5 \mathrm{~cm}$ in the lower third of the esophagus and at a location different from the first mass. A biopsy of this second mass confirmed intramucosal adenocarcinoma arising in Barrett's metaplasia and high-grade dysplasia (figure 2e). Endoscopic ultrasonography confirmed a lower third esophageal mucosal mass extending $35-40 \mathrm{~cm}$ from the incisor with no invasion of the muscularis propria or evidence of spread into the esophageal wall. There was no lymphadenopathy or evidence of distant metastases by CT scan, endoscopic ultrasonography and the positron emission 

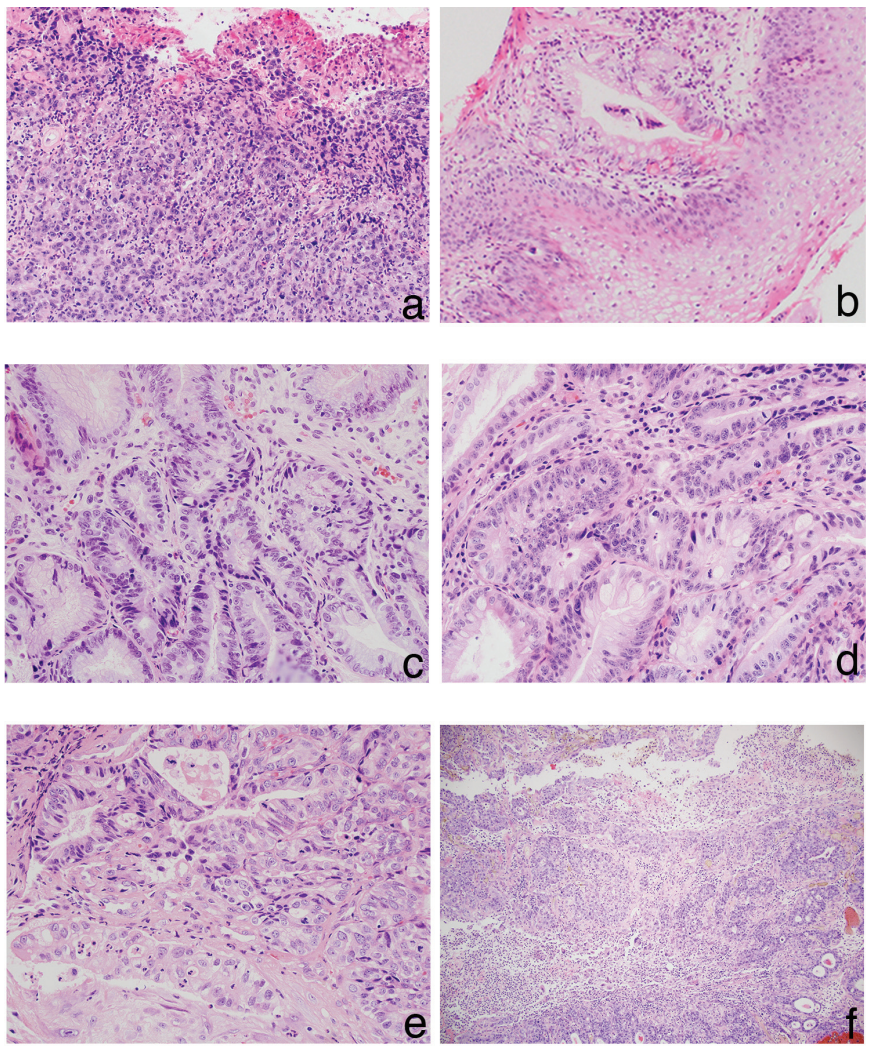

Figure 2. (a) Invasive poorly differentiated adenocarcinoma arising at the lower end of the esophagus. There is a solid proliferation of highly atypical epithelial cells with some gland formation. Note the surface ulcer with fibrinous exudate. (b) Postchemotherapy endoscopic biopsy (8 months after initial diagnosis). Cardiac-type mucosa with intestinal metaplasia, consistent with Barrett's esophagus. There is no evidence of dysplasia. Note the accompanying squamous re-epitheliazation. (c) Endoscopic biopsy of lower esophagus 15 months after completion of chemotherapy demonstrated high-grade dysplasia. The glands show architectural complexity (manifesting as crowding) with nuclear stratification and hyperchromasia. (d) Thirty-months postchemotherapy. High-grade dysplasia persists. (e) Three and one-half years after chemotherapy intramucosal adenocarcinoma was found in the background of high-grade dysplasia. The lower aspect of the figure shows an elongated nest of neoplastic cells invading the lamina propria. (f) Intramucosal adenocarcinoma in esophagectomy specimen. The high-grade dysplasia is accompanied by neoplastic cells infiltrating the lamina propria and muscularis mucosa. Despite extensive sampling of the lesion for histologic evaluation, there was no involvement of the submucosa.

tomography scan. The positron emission tomography scan, however, demonstrated uptake in the lower esophageal mass. The patient was diagnosed with a second de novo adenocarcinoma of the lower third of the esophagus, which was staged as cT1 N0 M0 (stage I) disease.

The patient subsequently underwent distal esophagectomy (up to the level of the azygous vein), esophagogastric anastomosis and pylorotomy by thoracoabdominal approach with periesophageal and perigastric lymph node dissection. Cervical esophagogastric anastomosis was not achievable due to the patient's obesity. Pathology demonstrated intramucosal adenocarcinoma with no submucosal, lymphatic, lymph node or vascular invasion, and was staged as pT1b N0 disease (figure 2f). The surgical margins of resection were free of malignancy but were involved by Barrett's metaplasia with low- and high-grade dysplasia. Because of the early stage of this second malignancy, no adjuvant therapy was recommended.

Subsequent follow-up for 24 months postsurgery with EGD has not demonstrated recurrence of Barrett's dysplasia or malignancy. He continues to have symptoms from gastroesophageal reflux disease (GERD) that is relieved by taking a proton pump inhibitor.

\section{Discussion}

Approximately 400,000 cases of esophageal cancer are diagnosed annually world wide. ${ }^{1}$ Of these approximately 14,250 are diagnosed in the United States with 13,570 people estimated to die from the disease in $2005 .^{2}$ However, the incidence of squamous cell esophageal carcinoma has decreased in the western hemisphere. ${ }^{7}$

The prognosis for esophageal cancer is dismal, although the 5 -year survival has modestly improved from $5 \%$ to $15 \%$ in the past three decades. Systemic metastatic disease is present in $50 \%$ of patients at the time of diagnosis, and the majority of the remaining group having localized regional disease at diagnosis will ultimately develop systemic metastases. ${ }^{8}$ However, 3-year survival rates range from $44 \%$ to $63 \%$ in patients with localized cancer (stage I and IIA) and from $6 \%$ to $10 \%$ in those with involvement of regional lymph nodes (stage IIB and III). ${ }^{9}$ The prognosis is extremely bleak in recurrent and advanced metastatic disease with most patients dying within 2 years in this stage. ${ }^{10}$ It is believed that chronic GERD exposes lower esophageal mucosa to gastric acid and bile, resulting in lower esophageal epithelium change from squamous to intestinal columnar type (metaplasia). Subsequently with genetic changes in P53 and P16, the epithelium becomes dysplastic and later may progress into malignancy. ${ }^{11-13}$

Ordinarily, locally advanced disease can cause dysphagia, anemia due to ulceration, weight loss, food sticking in esophagus, regurgitation and aspiration pneumonia, though our patient did not have any of these symptoms. Obesity, smoking, alcohol intake and GERD were his predisposing factors, and he presented with distant metastatic disease in the lymph nodes, lungs and liver.

Lower esophageal adenocarcinoma is commonly associated with Barrett's metaplasia, dysplasia and disease extension into the gastroesophageal junction. ${ }^{12}$ Although Helicobacter pylori is known to be present in gastric adenocarcinoma and gastric lymphoma, it is usually found with lower incidence in GERD. ${ }^{13-15}$ Current debate is taking place over whether eradication of $H$. pylori by antibiotics after treatment of peptic ulcer disease may be giving rise to increased incidence of GERD and Barrett's esophagus. ${ }^{15}$ 
Treatment options for advanced esophageal cancer have changed considerably over the past two decades. Initially surgery alone was the gold standard of treatment (5-year survival rate of $15 \%$ to $20 \%$ ) for early stage esophageal cancer. ${ }^{16}$ However, chemotherapy and radiotherapy, in both pre- and postoperative phases, have started to improve treatment outcomes. Radiation therapy in the adjuvant or neoadjuvant setting has been shown to reduce the incidence of local recurrence but does not provide any improvement in survival. ${ }^{16}$ Combined chemo-radiation as a definitive therapy has been demonstrated to be effective. ${ }^{16}$ The results of its use have been subjected to various randomized controlled trials comparing this with chemotherapy or radiation therapy alone, with or without surgery. The Radiation Therapy Oncology Group 8501 trial showed progression free survival is better with chemo-radiation as compared to radiation alone. ${ }^{17,18}$ The intergroup trial has shown that a higher dose of radiation does not improve survival in combination with chemotherapy. ${ }^{19}$ Use of neoadjuvant chemotherapy has shown variable results in different studies. A randomized study conducted by the Medical Research Council of the United Kingdom reported a significant benefit in overall survival for patients who received preoperative chemotherapy. ${ }^{20}$ A retrospective comparison of neoadjuvant chemo-radiation versus surgery alone showed dramatic improvement in survival in stage II or III lower esophageal and gastroesophageal junction adenocarcinoma with preoperative chemo-radiation. ${ }^{21}$ On the other hand, the North American Intergroup trial by Kelsen et al did not report any significant survival benefit with preoperative chemotherapy. ${ }^{22}$

Combined use of chemotherapy and radiation before surgery in locally advanced disease has been studied in various trials. An analysis of these studies shows that although there is no significant improvement in median survival, there is improved resectability, decreased local recurrence and better symptom relief. ${ }^{21,23} \mathrm{~A}$ study by Walsh et al, ${ }^{24}$ including only adenocarcinoma patients, found a significant improvement in survival with use of neoadjuvant chemo-radiation and surgery versus surgery alone.

For patients with metastatic disease who are considered incurable by surgery, palliative chemotherapy, radiation therapy and deployment of stents can markedly improve symptoms of dysphagia, pain and bleeding, and leads to improvement in functional quality of life. 10,16,25

We report a case of widely metastatic lower esophageal adenocarcinoma that was treated initially with chemotherapy. Single-agent chemotherapy with 5-fluorouracil, cisplatin/ carboplatin, paclitaxel, docetaxel, irinotecan, vinorelbine and vindesine can give rise to $15 \%$ to $35 \%$ variable responses and improvement of survival. A combination chemotherapy of 5 -fluorouracil plus cisplatin and paclitaxel gave 48\% responses. ${ }^{26}$ In our patient there was a dramatic, complete response to combination chemotherapy of 5-fluorouracil, paclitaxel and cisplatin/carboplatin chemotherapy with no endoscopic, radiologic or histologic evidence of cancer which was sustained for more than 4 years after initial diagnosis. Because of poor tolerance, cisplatin was replaced by carboplatin during later cycles. Serial endoscopies and biopsies continued to show Barrett's esophagus with evidence of dysplasia. Subsequent follow-up demonstrated progression of dysplasia and ultimately development of adenocarcinoma arising de novo from the dysplastic epithelium. There was no evidence of any distant metastases on the second occurrence. At this time the patient was treated as stage I cancer by distal esophagectomy and lymphadenectomy, and 2 years after surgery there is no evidence of recurrence of either of the two occurrences.

This case represents a unique combination of an initial excellent response to chemotherapy in widely metastatic disease followed by development of a second de novo primary adenocarcinoma, in a morphologically different location from the first tumor, due to persistence of Barrett's dysplastic changes. This case also emphasizes the importance of close follow-up of patients with Barrett's high-grade dysplasia with serial endoscopies and biopsies in order to establish an early diagnosis and definitive treatment.

A dysplastic change in Barrett's epithelium is a pre-malignant condition. It is possible Barrett's dysplasia and lower esophageal adenocarcinoma is increasing due to an increased incidence of GERD. Endoscopic surveillance at regular intervals may detect early stage, curable, malignant transformation of dysplasia. The effectiveness of aspirin, nonsteroidal antiinflammatory drugs and cyclo-oxygenase II inhibitors in decreasing the incidence of malignant transformation from dysplasia needs to be studied. ${ }^{4}$

\section{Acknowledgments}

The authors thank Marshfield Clinic Research Foundation for its support through the assistance of Alice Stargardt and Linda Weis in the preparation of this manuscript.

\section{References}

1. van Meerten E, van der Gaast A. Systemic treatment for oesophageal cancer. Eur J Cancer 2005;41:664-672.

2. Cancer Facts and Figures 2005 (American Cancer Society Publication), page 4. Available at: http://www.cancer.org/ downloads /STT/CAFF2005f4PWSecured.pdf. Accessed May 1, 2006.

3. Devesa SS, Blot WJ, Fraumeni JF Jr. Changing patterns in the incidence of esophageal and gastric carcinoma in the United States. Cancer 1998;83:2049-2053.

4. Souza RF, Spechler SJ. Concepts in the prevention of adenocarcinoma of the distal esophagus and proximal stomach. CA Cancer J Clin 2005;55:334-351.

5. Fitzgerald RC. Complex diseases in gastroenterology and hepatology: GERD, Barrett's, and esophageal adenocarcinoma. Clin Gastroenterol Hepatol 2006;3:529-537.

6. Brown LM, Swanson CA, Gridley G, Swanson GM, Schoenberg JB, Greenberg RS, Silverman DT, Pottern LM, Hayes RB, Schwartz AG, Liff JM, Fraumeni JF, Hoover Jr RN. Adenocarcinoma of the esophagus: role of obesity and diet. J Natl Cancer Inst 1995;87:104-109. 
7. Montgomery EA. Biopsy interpretation of the gastrointestinal tract mucosa. Philadelphia, PA: Lippincott Williams \& Wilkins; 2005.

8. Ilson DH. Oesophageal cancer: new developments in systemic therapy. Cancer Treat Rev 2003;29:525-532.

9. Hofstetter W, Swisher SG, Correa AM, Hess K, Putnam JB Jr, Ajani JA, Dolormente M, Francisco R, Komaki RR, Lara A, Martin F, Rice DC, Sarabia AJ, Smythe WR, Vaporciyan AA, Walsh GL, Roth JA. Treatment outcomes of resected esophageal cancer. Ann Surg 2002;236:376-384.

10. Shimada H, Kitabayashi H, Nabeya Y, Okazumi S, Matsubara H, Funami Y, Miyazawa Y, Shiratori T, Uno T, Itoh H, Ochiai T. Treatment response and prognosis of patients after recurrence of esophageal cancer. Surgery 2003;133:24-31.

11. Souza RF, Morales CP, Spechler SJ. Review article: a conceptual approach to understanding the molecular mechanisms of cancer development in Barrett's oesophagus. Aliment Pharmacol Ther 2001;15:1087-1100.

12. Weston AP, Banerjee SK, Sharma P, Tran TM, Richards R, Cherian R. p53 protein overexpression in low grade dysplasia (LGD) in Barrett's esophagus: immunohistochemical marker predictive of progression. Am J Gastroenterol 2001;96:1355-1362.

13. Eckardt VF, Kanzler G, Bernhard G. Life expectancy and cancer risk in patients with Barrett's esophagus: a prospective controlled investigation. Am J Med 2001;111:33-37.

14. Katz D, Rothstein R, Schned A, Dunn J, Seaver K, Antonioli D. The development of dysplasia and adenocarcinoma during endoscopic surveillance of Barrett's esophagus. Am J Gastroenterol 1998;93:536-541.

15. el-Serag HB, Sonnenberg A. Opposing time trends of peptic ulcer and reflux disease. Gut 1998;43:327-333.

16. Koshy M, Esiashvilli N, Landry JC, Thomas CR Jr, Matthews RH. Multiple management modalities in esophageal cancer: combined modality management approaches. Oncologist 2004;9:147-159.

17. Herskovic A, Martz K, al-Sarraf M, Leichman L, Brindle J, Vaitkevicius V, Cooper J, Byhardt R, Davis L, Emami B. Combined chemotherapy and radiotherapy compared with radiotherapy alone in patients with cancer of the esophagus. N Engl J Med 1992;326:1593-1598.

18. Cooper JS, Guo MD, Herskovic A, Macdonald JS, Martenson JA Jr, Al-Sarraf M, Byhardt R, Russell AH, Beitler JJ, Spencer S, Asbell SO, Graham MV, Leichman LL. Chemoradiotherapy of locally advanced esophageal cancer: long-term follow-up of a prospective randomized trial (RTOG 85-01). Radiation Therapy Oncology Group. JAMA 1999;281:1623-1627.

19. Minsky BD, Pajak TF, Ginsberg RJ, Pisansky TM, Martenson J, Komaki R, Okawara G, Rosenthal SA, Kelsen DP. INT 0123 (Radiation Therapy Oncology Group 94-05) phase III trial of combined-modality therapy for esophageal cancer: high-dose versus standard-dose radiation therapy. J Clin Oncol 2002;20:1167-1174.

20. Law S, Wong J. Therapeutic options for esophageal cancer. J Gastroenterol Hepatol 2004;19:4-12.

21. Rosato EL, Daluvoy SV. Neoadjuvant chemoradiotherapy with 5 fluorouracil (5FU), carboplatinum (C) and paclitaxel (T) dramatically improves survival in patients with clinical stage 2 and 3 adenocarcinoma of the distal esophagus and GE junction compared to surgery alone. J Clin Oncol (Meeting Abstracts) 2005;23:4204. Available at: http://meeting.jco.org/cgi/gca?gca=23\%2F16_suppl\%2F4204 \&sendit $=$ Get + All + Checked + Abstract $\% 28 \mathrm{~s} \% 29$. Accessed May 2, 2006.

22. Kelsen DP, Ginsberg R, Pajak TF, Sheahan DG, Gunderson L, Mortimer J, Estes N, Haller DG, Ajani J, Kocha W, Minsky BD, Roth JA. Chemotherapy followed by surgery compared with surgery alone for localized esophageal cancer. N Engl J Med 1998;339:1979-1984.
23. Shaheen NJ. Advances in Barrett's esophagus and esophageal adenocarcinoma. Gastroenterology 2005;128:1554-1566.

24. Walsh TN, Noonan N, Hollywood D, Kelly A, Keeling N. Hennessy TP. A comparison of multimodal therapy and surgery for esophageal adenocarcinoma. N Engl J Med 1996;15:335:462-467.

25. Shah MA, Kelsen DP. Combined modality therapy of esophageal cancer: changes in the standard of care? Ann Surg Oncol 2004;11:641-643.

26. Ilson DH, Ajani J, Bhalla K, Forastiere A, Huang Y, Patel P, Martin L, Donegan J, Pazdur R, Reed C, Kelsen DP. Phase II trial of paclitaxel, fluorouracil, and cisplatin in patients with advanced carcinoma of the esophagus. J Clin Oncol 1998;16:1826-1834.

\section{Author Affiliations}

Hemender S. Vats, MD, Department of General Internal Medicine, Marshfield Clinic, 1000 North Oak Avenue, Marshfield, WI 54449

\author{
Tarit K. Banerjee, MD, FACP, Department of \\ Hematology/Oncology, Marshfield Clinic, 1000 North Oak \\ Avenue, Marshfield, WI 54449
}

Jeffrey Resnick, MD, Department of Pathology, Marshfield
Laboratories, 1000 North Oak Avenue, Marshfield, WI 54449

Qaseem Khan, MD, Department of Hematology/Oncology, Marshfield Clinic, 1000 North Oak Avenue, Marshfield, WI 54449 